\title{
Variations
}

Variations

Revue internationale de théorie critique

$20 \mid 2017$

Expériences oppositionnelles

\section{La saturation du temps social}

Éléments de réflexion sur les conditions de possibilité d'un droit au temps comme modalité d'un droit à la ville

\section{Simon Le Roulley}

\section{(2) OpenEdition}

12 Journals

\section{Édition électronique}

URL : http://journals.openedition.org/variations/863

DOI : 10.4000/variations.863

ISSN : 1968-3960

Éditeur

Les amis de Variations

Référence électronique

Simon Le Roulley, «La saturation du temps social », Variations [En ligne], 20 | 2017, mis en ligne le 25 avril 2017, consulté le 25 février 2021. URL : http://journals.openedition.org/variations/863 ; DOI : https://doi.org/10.4000/variations.863

Ce document a été généré automatiquement le 25 février 2021.

Les ami•e•s de Variations 


\title{
La saturation du temps social
}

\author{
Éléments de réflexion sur les conditions de possibilité d'un droit au \\ temps comme modalité d'un droit à la ville
}

\author{
Simon Le Roulley
}

1 Il est aujourd'hui courant de lire différents travaux en sciences humaines et sociales abordant à nouveau la vieille thématique philosophique et anthropologique du temps. En effet, le problème du temps traverse les travaux de la sociologie française, de sa fondation par Durkheim jusqu'aujourd'hui. Durkheim, dans Les formes élémentaires de la vie religieuse ${ }^{1}$ l'évoquait déjà en reliant la problématique du temps aux espaces et à l'institution à partir des catégories de profane et sacré. Mauss, et peut-être surtout Hubert, se sont inscrits dans le continuum durkheimien en interrogeant la représentation du temps dans la religion et la magie ${ }^{2}$. Plus tard, toujours chez les durkheimiens, le temps sera étudié à travers la mémoire par Maurice Halbwachs ${ }^{3}$. Mais c'est surtout au lendemain de la guerre, par la rencontre de la tradition durkheimienne et du marxisme, que la problématique du temps va jalonner l'histoire des différents sous champs de la sociologie. Gurvitch et ses "temporalités explosives " ${ }^{4}$, Friedmann ${ }^{5}$ et Naville ${ }^{6}$ et les impacts de l'organisation scientifique du travail sur le temps de travail et les autres temps de la vie, Morin et son Esprit du temps ${ }^{7}$, tous fondent un socle légitimant le temps comme préoccupation majeure d'une sociologie française riche de la multiplicité des influences qui la traversent. L'impact du structuralisme et du panstructuralisme va néanmoins tempérer l'intérêt porté au temps en sociologie et plus généralement à l'approche sociohistorique des institutions ${ }^{8}$. Mais, bien qu'à la marge et réunis sous l'égide de William Grossin, les «Temporalistes" vont s'inscrire dans ce continuum à leur tour ${ }^{9}$. Néanmoins, l'histoire de la sociologie est largement écrite par ses vainqueurs, ceux qui ont paradoxalement évacué la dimension historique de la sociologie.

2 Pour autant, depuis une dizaine d'années le spectre du temps semble hanter à nouveau le paysage sociologique. Du désormais classique de Hartmut Rosa ${ }^{10}$ jusqu'à Jean-Pierre Boutinet $^{11}$, en passant par Michel Lallement puis Patrick Cingolani ${ }^{12}$, tout porte à croire qu'un regain d'intérêt pour la thématique du temps et des temporalités s'est imposé aux chercheurs, au-delà de la sociologie du travail qui a maintenu le temps comme 
modalité de ses enquêtes. Et pour cause, la question du temps plane sur le quotidien des individus. Que ce soit en positif par la diminution du temps de travail et la précarisation de celui-ci, ou en négatif, par des initiatives qui tendent, quoique parfois inconscientes, à mettre en place des temps communs autour d'espaces communs, comme les jardins partagés, les lieux d'activités collectifs, ou les luttes antiproductivistes qui interrogent les rythmes de production ${ }^{13}$.

3 Par ailleurs, de récents évènements politiques conduisent à interroger radicalement ce rapport au temps. Alors qu'historiquement les gauches ont lutté pour la diminution du temps de travail, l'actuel ministre de l'Économie E. Macron, bien que se justifiant d'une défense théorique du cadre légal de la durée du travail limitée à 35 heures hebdomadaires, confesse qu'il lui paraitrait envisageable qu'une certaine "souplesse » soit mise en place et que la durée du travail puisse être négociée entre employeurs et salariés ${ }^{14}$. D'un point de vue socio-anthropologique, on observe, par cette décentralisation vers l'échelle locale des modalités d'encadrement du temps de travail, une tendance possible à une individualisation des emplois du temps ${ }^{15}$. Ce processus est déjà en cours et n'est qu'une poursuite de la dépersonnalisation et de l'individualisation du travail qu'inscrit la rationalisation de l'organisation du travail, décrit depuis Friedmann jusqu'à Boltanski. Et bien qu'une sociologie héritière de Touraine maintient que les luttes autour du travail se sont affaiblit au profit de nouveaux mouvement sociaux, force est de constater que le mouvement contre la « loi Travaille !» du printemps 2016 semble avoir réveillé le fantôme de la lutte des classes notamment à partir d'une critique de l'individualisation et de la décentralisation des négociations concernant les conditions de travail, conditions qui s'avèrent nécessairement temporelles. Dans une société où le temps de travail rythme encore largement les autres temps de la vie, on peut se poser la question des effets socioanthropologiques de cette individualisation du rapport au temps. Jonathan Crary ${ }^{16}$ montrait dans un livre récent les effets d'une ville ouverte 24 heures sur 24. Quel autre espace que l'urbain pourrait mieux s'adapter à des emplois du temps désynchronisés ? Ces phénomènes nous conduisent à défendre l'hypothèse de la production d'un temps et d'un espace saturés comme corolaire d'une crise institutionnelle qui a pour conséquence l'effacement progressif des cadres sociaux de la vie commune.

Avant d'expliciter plus en avant cette hypothèse, de décrire ses causes et ses effets, il convient de rappeler un certain nombre d'éléments épistémologiques permettant de comprendre la démarche de recherche qui consiste à étudier le temps en relation étroite avec l'espace urbain, le travail et la vie quotidienne. Dans un premier temps nous présenterons l'approche qui tente d'aborder le temps comme un phénomène institutionnel puis, dans le même mouvement, la façon dont la ville, le travail et la vie quotidienne permettent d'accéder à ce phénomène difficilement dicible et visible. Dans un second temps, nous présenterons les causes profondes et les conséquences de la saturation du temps social et de l'espace social, ces deux derniers étant à considérer comme des « produits» dans la droite ligne des réflexions tracées par Henri Lefebvre ${ }^{17}$. Ce sera enfin l'occasion, dans une sociologie critique impliquée de l'émancipation, de baliser des pistes qui conduisent à l'établissement d'un droit au temps qui résonne au droit à la ville sur le principe du commun, à partir de cas figuratifs qui nous semblent interroger radicalement le droit au temps autant que le droit à la ville ${ }^{18}$. Saisir le temps social dans une sociologie critique : l'approche « durkheimo-marxiste » 
5 Dans ses cours sur l'État au Collège de France, Pierre Bourdieu rappelle que l'État a pris le relais de l'Église dans la structuration de l'ordre social à travers la synchronisation du temps ${ }^{19}$. Les historiens nous ont enseigné les évolutions du temps social et sa structuration au cours de l'histoire, la façon dont il révèle à la fois un mode de production, un ordre politique, une organisation des routines quotidiennes et l'espace social traversé par ces dimensions. Au mode de production agraire et artisanal des sociétés précapitalistes soumises aux scansions saisonnières et à l'alternance des jours et des nuits s'est substitué un temps discipliné qui apparaît avec l'érection de l'État moderne et du développement des manufactures puis de l'industrialisation ${ }^{20}$. L'Église a longtemps incarné le pouvoir politique qui imposait le temps social ${ }^{21}$, marquant par le son des cloches les heures de la mise au travail et du repos retrouvé à l'échelle du quotidien, mais aussi les fêtes qui rythmaient les cycles saisonniers, qui conservait une " cohérence " avec un temps cyclique lié à la soumission de l'homme à la nature et à ses rythmes et laissait entrevoir une certaine porosité entre les différents temps de la vie ${ }^{22}$. C'est sous la poussée de la rationalisation du pouvoir et du travail que le temps social deviendra, selon les mots de Thompson, un «temps discipliné », en ce sens que les temps de la vie vont se structurer par l'encadrement d'un temps de travail qui donne le rythme de la vie quotidienne. La discipline qui s'impose par le travail industriel s'étend donc à l'ensemble de la vie quotidienne et se trouve relayé plus encore par l'architecture des espaces de travail et des espaces de l'action institutionnelle comme l'école ${ }^{23}$. Mais dans un XIXe siècle qui glorifie le libéralisme, l'attention des dominants se porte sur le développement de l'industrie et la solidification des institutions encore récentes et fragiles, ce qui passe par la rationalisation de l'espace urbain dans son ensemble afin d'éviter toute entreprise populaire de déstabilisation du régime politique - expériences dont le Paris de Napoléon III et Hausmann fut le terrain privilégié2 ${ }^{24}$. L'apologie de la propriété privée fait de la ville un véritable lieu d'investissement, un espace où chaque propriétaire est quelque part un entrepreneur. Aux vieilles conventions du passé se substituent une liberté d'entreprendre, c'est-à-dire de produire de l'immobilier comme on l'entend sans prendre en considération l'harmonie, sans tenir compte non plus des désirs des habitants : l'espace se privatise, la sphère publique n'intervient plus ou presque. C'est dans ce contexte que fleurissent, aux abords des usines des maisons précaires dans lesquelles se logent les ouvriers ${ }^{25}$. L'espace public bourgeois - en ce qu'il est produit par la bourgeoisie, classe de propriétaires oisifs - ségrègue et rassembleen des lieux et des intérêts communs la classe dominante, pendant que s'agglutinent autour des usines les familles ouvrières ${ }^{26}$. Ce phénomène que Lefebvre nomme "implosion-explosion " ${ }^{27}$, c'est le rapport dialectique entre cette immense concentration dans la ville (capitaux, individus, activités, culture, etc.) et la projection en fragments distincts de certaines catégories sociales dans les périphéries, banlieues, centre, etc. Cette nouvelle réalité urbaine traduit la société de classe qui voit le jour et qui s'impose par la disciplinarisation de l'espace de la ville et du temps de travail. La dépossession de l'espace n'est donc pas un phénomène neutre, mais s'avère être, comme le disait Lefebvre, la production d'un espace qui reflète les rapports de force et participe à la reproduction des rapports sociaux de domination et d'exploitation. De la même manière, si la durée du temps de travail s'avère être une revendication historique du mouvement ouvrier, les premières revendications furent portées par les patrons pour imposer un encadrement du temps de travail qui empêche la fuite vers les champs (Freyssinet, 1998). Alors qu'une certaine porosité entre temps de travail et vie quotidienne était perceptible avant 
l'industrialisation, le morcellement des temps va s'opérer conjointement à la distinction entre le rural et l'urbain ${ }^{28}$, l'un représentant le progrès et le mode sociétaire, l'autre la tradition et le communautaire. La fixation des ouvriers à l'usine a en effet pour conséquence de réduire les mobilités des ouvriers de l'urbain vers le rural. À la politique de l'espace s'adjoint une politique du temps qui cartographie la domination capitaliste en introduisant des représentations sociales qui isolent le mode de vie urbain du mode de vie rural, et qui, au sein de la vie urbaine distinguent les cultures nobles - celles du loisir de consommation ostentatoire - de l'ignoble, la culture ouvrière encore largement imprégnée des mœurs rurales ${ }^{29}$.

6 C'est dans ce contexte que la sociologie va s'ériger. Deux sociologies nous intéresseront particulièrement ici en ce qu'elles proposent une méthode qui nous semble la plus adéquate à l'explication des fondements sociaux du temps social. La sociologie de Durkheim propose en effet contre une philosophie trop spéculative d'observer la société telle qu'elle est. Il s'agit pour lui de proposer un objet et une méthode à la sociologie pour lui conférer le statut de science, le fait social, c'est-à-dire les manières d'agir, de penser et de sentir, extérieures à l'individu et qui s'imposent à lui ${ }^{30}$. Ces forces qui guident nos conduites nous apparaissent comme naturelles nous explique-til, sauf lorsqu'on leur résiste. C'est que les faits sociaux nous préexistent, nous traversent, et échappent à notre conscience. Afin d'expliquer le fait social, Durkheim propose donc de définir la sociologie comme «la science des institutions ${ }^{31}$, de leur genèse et de leur fonctionnement $»^{32}$ et de chercher les "causes profondes qui échappent à la conscience $»^{33}$. Bien que différant sur la forme de l'engagement, la sociologie de Marx rejoint en certains points celle de Durkheim. Pour Marx en effet, « ce n'est pas la conscience des hommes qui détermine leur être ; c'est inversement leur être social qui détermine leur conscience $\|^{34}$, c'est-à-dire que des forces objectives s'imposent à l'individu. Marx montre également, comme Durkheim, l'apparente naturalité des faits sociaux qui repose sur une production collective dont on trouve la genèse dans l'histoire. L'État comme institution incarne l'historicité, il cristallise dans sa fonction reproductive des rapports sociaux les rapports de force historiques. Aux mains des dominants, le maillage institutionnel lui permet de reproduire par l'appropriation du savoir légitime un ordre injuste qui apparaît dès lors comme naturel ${ }^{35}$. C'est ainsi que la Commune de Paris, parce qu'elle décrète l'appropriation de la ville par le peuple dominé et érige des institutions décentralisées, représente pour Marx une expérience historique et un modèle du renversement de la table ${ }^{36}$. Cela dit, il ne faut pas aller jusqu'à dire qu'il $\mathrm{y}$ a une géographie sociale ou une sociologie spatialisée des rapports sociaux chez les deux auteurs ${ }^{37}$, mais il y a un intérêt pour la projection dans l'espace de formes sociales concrètes qui doit être approchée de manière sociohistorique et une relation intime entre le spatial et le temporel. Pour Marx, le procès de circulation de la valeur provoque une suppression de l'espace par le temps ${ }^{38}$. Marx rate ici sans doute, comme le montre David Harvey, que ce n'est pas tant une annihilation de l'espace par le temps, que la production d'autres espaces qui correspondent à la dynamique d'accélération du capitalisme ${ }^{39}$. Reste que, d'une manière inédite, l'œuvre de Marx met en avant le double problème temporel du capitalisme : l'organisation de la dépossession du temps de l'ouvrier par le salariat ${ }^{40}$; l'hypothèque de l'avenir par spéculation ${ }^{41}$. De son côté, Durkheim aborde la problématique de l'espace et, malgré un certain évolutionnisme qu'on lui connaît, dessine les prémisses d'une critique d'une "autonomie relative de l'espace ${ }^{42}$ en insistant sur le fait qu'au-delà des différences géomorphologiques de l'espace physique 
on peut observer des types sociaux identiques : «Si, cette fois, la société tient au sol, ce n'est pas parce qu'elle a subi son action, mais au contraire parce qu'elle se l'est assimilé. C'est lui qui porte sa marque, loin qu'elle se modèle sur lui. Ce n'est donc plus la terre qui explique l'homme, mais l'homme qui explique la terre $»^{43}$.

7 Il n'est alors pas étonnant, malgré ces manques, d'observer plus tard dans l'histoire de la sociologie française, un intérêt particulier porté à la dimension spatiale et temporelle du social. C'est aussi que, au cours de l'histoire, la diminution du temps de travail va modifier le rapport au temps et avoir une incidence sur la production de l'espace urbain à différentes échelles. La rationalisation du travail a contribué à sa dépréciation, et c'est désormais dans le quotidien que les ouvriers cherchent à valoriser leur activité. Obtenus en 1936, les congés payés ne sont pas uniquement une victoire du mouvement social, mais un compromis politique censé ouvrir la relance de l'économie ${ }^{44}$. Mais aux vieilles images des vacanciers sur les routes vers la mer, il faut leur substituer une réalité sociologique moins glamour. Les ouvriers n'avaient pas réellement les moyens économiques pour accéder aux activités nobles du loisir avant les années $1950^{45}$, ni même les cadres de références leur permettant d'être à l'aise dans des centres-ville bourgeois. Le loisir ouvrier, ce que Dumazedier appelait le semi-loisiri ${ }^{46}$, consiste plutôt en une forme de travail en dehors de l'usine, travail qui peut être domestique comme le bricolage, ou encore comme une seconde activité de complément de revenu. Le gouvernement mettra donc en place un encadrement du loisir qui s'inscrit dans la continuité des politiques des mœurs évoquées déjà par Tocqueville et travaillées par Hausmann qui vise la pacification sociale. Le développement du loisir ouvrier s'appuie principalement par le développement des sports et des équipements publics urbains ${ }^{47}$. La politique du sport de masse passe à la fois par le développement de l'accès au sport et par sa mise en spectacle, figuration de la force des corps que le travail standardisé ne permet plus de présenter ${ }^{48}$. Il y a donc ici aussi une imposition politique d'espaces produits pour un temps particulier lui aussi largement déterminé par le système institutionnel. La demande de loisir, même si elle a un caractère a priori spontané devient un secteur de la vie quotidienne que «l'organisation sociale vient orienter, préciser, infléchir, modifier dans les satisfactions qu'elle lui offre $»^{49}$ en créant une ingénierie des besoins permettant de dissocier ce que Lefebvre nomme le «besoin général de loisir» - corollaire du travail parcellaire - et les «besoins concrets différenciés »- les besoins qui s'expriment à travers la production industrielle du loisir. Ce que l'on nomme la culture de masse se situe dans cette dialectique du général et du particulier, de l'homogène et de la différence.

\section{Des espaces-temps comprimés aux espaces-temps saturés}

L'accès au temps libre et son encadrement, s'ils participent d'une homogénéisation culturelle, produisent un espace urbain fragmenté qui crée un emploi du temps qui se matérialise dans le proche. Dumazedier note d'ailleurs que si la fonction de développement (temps pour la contemplation, l'activité libre ou l'action désintéressée) peut exprimer une résistance au travail monotone par des semis-loisirs comme le bricolage, la fonction de divertissement (fuite du sentiment de privation et de dépossession) peut quant à elle entrainer un désintérêt non seulement vis-à-vis de l'activité de travail, mais plus généralement vis-à-vis de ce qu'est le travail et son mode 
d'organisation et donc des solidarités. La valorisation du loisir ne dévalorise pas seulement le travail, mais le rend essentiellement inintéressant, de sorte que l'envie même d'améliorer ses conditions de travail s'éloigne dans l'en-dehors sans que le corps ne quitte l'usine, il s'en divertit, s'en détourne simplement, si bien qu'on en oublie le fait que la vie quotidienne reste dominée par le temps de travail en ce que celui-ci détermine légalement et quantitativement les autres temps de la vie. L'accroissement et la valorisation du temps libre ont pour conséquence un effacement du caractère discipliné du temps social, effacement qui ne suppose pas pour autant une libération, mais une mutation de la vie quotidienne qui ne fournit « non plus seulement un temps de repos et de récupération, mais un temps de consommation $»^{50}$ Cette dynamique sociale que l'on a appelée « société de consommation » s'appuie donc sur une politique du temps et une politique de l'espace.

9 La politique du loisir entraîne, comme l'ont montré les historiens, une rationalisation $\mathrm{du}$ loisir. Si les ouvriers partent peu et se recentrent sur les associations ouvrières, celles-ci, après la libération, organiseront de plus en plus de départs loin des quartiers d'usines pour rompre avec le temps et l'espace du travail. Mais paradoxalement, Richez et Strauss montrent que ces séjours sont organisés selon un emploi du temps précis, même si la liberté de participer aux activités était accordée. Dans un article célèbre, Henri Raymond révèle le paradoxe spatio-temporel de ces voyages organisés. À la fois on observe un éloignement du temps et de l'espace de travail. Plus encore peut-être, d'après Raymond, on y voit une inversion : au temps long, linéaire et chronométré du travail s'oppose un temps court, réduit; à la pratique sociale en des espaces réduits à l'échelle des quartiers, l'espace vécu s'élargit ${ }^{51}$. Néanmoins, on conserve dans ce loisir d'après-guerre un rapport au temps mesuré de l'économie qui ne tardera pas à substituer, au loisir ouvrier des associations et organisations syndicales reposant sur un certain solidarisme, une pratique consommatoire. L'avènement du loisir a permis la création d'un temps pour soi, mais ce temps reste soumis à la poussée rationaliste issue du travail ${ }^{52}$. Et à mesure que le temps des vacances libère dans ces villages estivaux, la vie quotidienne urbaine se "comprime» autour du travail et de l'habitat. La dynamique fonctionnaliste accentue ce phénomène par la production d'espaces spécialisés qui permettent à la fois de réduire l'espace vécu en rapprochant les différentes «fonctions » de l'urbain dans le proche. Ceci participe d'une rationalisation de la relation espace et temps, en ce sens qu'il faut moins de temps pour effectuer ses activités à mesure que l'espace vécu est réduit. Le fonctionnalisme, Le Corbusier en tête, est obsédé par l'ordre et cherche à lutter contre la perte de temps, comme Taylor et Ford l'on fait en mettant en place les procès d'organisation scientifique du travail ${ }^{53}$. À la standardisation des tâches qui convoquaient déjà un sentiment de substituabilité chez les ouvriers s'ajoute une standardisation de «l'habitat». L'urbanisme fonctionnaliste s'appuie, selon la Charte d'Athènes, sur quatre grands principes que sont l'habitat, le travail, la récréation et la circulation entre ces différentes activités. La reconstruction participera à l'imposition de ce nouvel urbanisme qui, dans le développement des banlieues, produit des espaces clos sur eux-mêmes. La ville est alors un dispositif qui repose sur une planification abolissant les anciennes formes de vie et ségréguant toujours plus l'espace des riches et celui des pauvres ${ }^{54}$. Le "métalangage " $\mathrm{du}$ fonctionnalisme circule dans les discours des films du ministère de reconstruction et de l'urbanisme qui promeut le fonctionnel et l'idée de se défaire de l'archaïsme des villes polycentriques et polyfonctionnelles ${ }^{55}$ et où la circulation des hommes est pensée par la vitesse, c'est-à-dire par le développement de larges routes pour la circulation 
automobile, circulation de la force de travail et des marchandises ${ }^{56}$. Standardiser, homogénéiser l'habitat ne signifie pas créer de l'identique, mais faire de l'espace, des volumes immobiliers et du sol, des biens interchangeables et donc échangeables. La politique de l'espace tend à homogénéiser sous le signe du quantitatif, comme le temps se mesure déjà en argent.

10 Mais cet urbanisme produit également de la monotonie. La part de liberté individuelle inhérente à la production des routines est largement dominée par l'espace "produitproducteur $\aleph^{57}$ qui influe sur les conduites. L'impossible appropriation et l'entrave à des routines originales qui conduit à des routines standardisées que l'expression " métroboulot-dodo" vulgarise habilement. Bien que le mot routine puisse avoir une connotation négative dans le sens commun, il incarne d'un point de vue sociologique une liberté, en ce sens qu'il traduit une contrainte quotidienne auto-administrée permettant de "baliser" son quotidien ${ }^{58}$. Si le mode de vie s'homogénéise par la socialisation que le système institutionnel met en place et les impératifs du mode production, nous conservons une liberté relative dans l'organisation de notre vie quotidienne, ce que Durkheim déjà analysait : «Il n'est pas de conformisme social qui ne comporte toute une gamme de nuances individuelles. Il n'en reste pas moins que le champ des variations permises est limité ${ }^{59}$. Pour le sociologue de la vie quotidienne, les routines permettent de dégager du temps par l'auto-organisation des activités les plus quotidiennes et la mise en place d'un emploi du temps individuel qui s'applique dans l'espace vécu de la vie ordinaire. Cette autocontrainte «libère d'avoir à décider trop souvent » et permet « de rationaliser la vie pour accumuler les activités, mais aussi de penser à autre chose, de s'évader, voire de créer " ${ }^{60}$, car "l'invention ne peut prendre son essor que sur un terrain soigneusement reconnu et balisé $»^{61}$. Mais l'urbanisme fonctionnaliste par le zonage réduit à la fois cette liberté en produisant des espaces assignés précisément à des séquences de temps, et réduit la possibilité du hasard par une orthogonalité qui infléchit les mobilités à l'économie de temps, principe essentiel dans la sphère de la production capitaliste qui domine désormais l'espace et les temps de la vie. De plus, la division fonctionnelle se joue à une autre échelle et confère à certaines villes ou régions des spécificités en matière de production qui permettent d'éclater les classes populaires sur le territoire en les désolidarisant en fonction des métiers, puis à l'échelle locale, par quartier. L'urbanisation capitaliste repose ainsi sur la « réduction du coût et du temps de mouvement dans l'espace », dans la construction d'infrastructures permettant l'accélération de la circulation et par l'appropriation du capital foncier, et enfin à travers une organisation territoriale, c'està-dire une mise en ordre des choses et des êtres qui permet la reproduction des rapports de production ${ }^{62}$.

11 Les critiques artistiques politiques de la routine qui s'inscrivent sur les murs des villes traduisent un rejet d'une quotidienneté de plus en plus appauvrie et limitée. Du « changer la vie » des surréalistes jaillira le « changer la ville» des situationnistes. On avait pu voir, dans l'histoire, les classes ouvrières s'éloigner des préoccupations du travail pour se concentrer sur un loisir divertissant. Mais lorsque la consommation devient plus austère, que la quotidienneté s'épuise dans un répétitif artificiel, les vieilles habitudes reprennent du service. C'est pour éviter un nouveau mai 1968 que la question urbaine va être si importante dans les années 1970, années de crise économique. En effet, Valérie Giscard d'Estaing décide d'arrêter la construction des grandes tours fonctionnalistes en 1973 de peur que les grands ensembles ne se "ghettoïsent». La nouvelle politique urbaine, décryptée minutieusement par Jean- 
Pierre Garnier et Denis Goldschmidt, repose sur la réhabilitation du bâti existant et le développement de services publics de proximité dans les quartiers populaires. Reprenant à leur compte certaines thèses du droit à la ville, le président et ses ministres cherchent à réhabiliter les espaces de sociabilités au sein des quartiers, à revaloriser les centres historiques comme espace d'identification des citadins en y développant des activités de commerce et de culture, à rendre l'espace plus polyfonctionnel, développer les transports en commun, ressusciter les fêtes et célébrations, et revitaliser la démocratie locale ${ }^{63}$. C'est l'abandon de l'utopie de la villemachine. En pleine crise, c'est aussi un bon palliatif qui permet de cibler les causes de la misère comme résultantes d'un espace à réhabiliter plutôt que de penser les ressorts économiques et politiques de la crise. Dans ce climat, le virage qualitatif de l'aménagement repose également sur un projet visant à prévenir «l'apparition de plus en plus fréquente de formes de luttes [...] illégales et extra-institutionnelles " ${ }^{64}$. La nouvelle politique urbaine tend donc à faire apparaitre les problèmes de la vie quotidienne comme des résultats d'un problème d'organisation locale de l'espace, tandis que les problèmes économiques trouveraient leurs causes à l'échelle de l'espace national. La réhabilitation des équipements collectifs permet en outre moins la reproduction de la force de travail que la reproduction des rapports sociaux en incitant à la participation locale, et vise à réduire les inégalités de capitaux culturels tout en maintenant des inégalités de classes ; c'est-à-dire à créer au niveau local que la vie peut changer sans changer les structures sociales, en promouvant une "participation active " et une sociabilité qui se résout par la communauté de quartier plus que par des solidarités et un engagement émergeant d'une conscience de classe ${ }^{65}$. Ce n'est alors plus le sentiment d'une commune expérience de dépossession du temps qui fonde le collectif en abolissant les frontières - des murs des usines aux frontières nationales -, mais l'espace de l'habiter, un espace conçu et administré pour la paix sociale.

\section{Droit à la ville et droit au temps}

12 Que dire désormais du présent? Ce rétrospectif sociohistorique tend à faire apparaître la façon dont l'espace et le temps sont agis par des déterminismes institutionnels et économiques, et propose une lecture du capitalisme comme politique de l'espace et du temps. On voit alors dans l'histoire les liens qui unissent ces deux dimensions fondamentales de la sociologie et de la géographie sociale. On voit également des processus d'absorption des contradictions, la façon dont le capitalisme contient en lui une logique destructrice-créatrice, c'est-à-dire comme l'explique Harvey qu'il produit des espaces sur les ruines et que, au moment venu, ces espaces eux-mêmes seront amenés à disparaître sous la poussée des contradictions. La nouvelle politique urbaine menée par Valérie Giscard d'Estaing annonce un retour du qualitatif dans l'aménagement urbain et une relocalisation des problèmes politiques. L'analyse de Goldschmidt et Garnier démystifie les pseudo velléités émancipatoires qui présideraient à ce "virage à gauche", en expliquant qu'il s'agit avant tout d'une nécessité historique de pacification des rapports sociaux liée à la crise économique qui a conduit à cette stratégie pour focaliser l'attention et capter la participation au niveau local, désolidarisant ainsi les classes populaires et décentrant le discours revendicatif. La politique locale et participative existe encore aujourd'hui, des conseils de la vie locale aux désormais célèbres grands débats publics ${ }^{66}$, et continue de proposer des modèles consultatifs évitant toute conflictualité exprimant les antagonismes sociaux. 
La stratégie localiste et participative s'est étendue dans différents champs. Au niveau du travail par exemple, elle a été appropriée par les théoriciens du management qui n'hésitent pas à appliquer une architecture hiérarchique polyfonctionnelle de l'entreprise, à exploser les métiers en des services mixtes et à simuler du jeu, du fun pour créer du collectif qui ne repose pas tant sur les conditions de travail que sur les objectifs planifiés à remplir, travaillant ainsi à la manière d'une ingénierie des désirs à enrôler les salariés par les affects ${ }^{67}$. De plus, la sous-traitance implique des réalités statutaires différenciées au sein des mêmes équipes, et la peur du chômage accroît la mobilité des salariés. Cet éclatement des situations de travail et des conditions de vie freinent la syndicalisation. Les conséquences temporelles qui en résultent s'expriment dans des rapports individualisés au quotidien, à des rythmes différenciés d'un salarié à l'autre: si l'espace de travail est partagé, l'expérience du temps ne l'est pas ${ }^{68}$. Cette organisation du travail répond à un esprit de dépolitisation de la culture d'entreprise afin de déplacer sur "le bon sens" et le ludique des revendications salariales qui $s^{\prime}$ individualisent ${ }^{69}$. Si le travail reste le temps dominant, le temps structurant la vie quotidienne, l'individualisation du temps de travail introduit par l'idéologie de la flexibilité, au travail précarisé et l'ouverture aux heures supplémentaires, ne lui permet plus d'être un temps structurant collectivement, mais seulement individuellement. Pas étonnant alors d'observer une tendance à l'ouverture des villes vingt-quatre heures sur vingt-quatre et sept jours sur sept, afin qu'elles s'adaptent à la dysrythmie du travail. La précarité et l'emploi par vacations ou par missions placent les précaires dans une situation de dépendance et gêne la création de routines puisque chacun est dans l'attente. Dans les espaces de travail, on peut observer des réalités différentes entre les stables et les instables, qui rendent les sociabilités plus complexes et décentrent voire entravent des solidarités ${ }^{70}$. Situation de décalage entre des référentiels anciens et nouveau du rapport au temps et au travail, des conflits liés aux pratiques quotidiennes peuvent éclater, et sont traversés par les dimensions spatiales, temporelles et normatives. Ce n'est pas tant que le temps "manque ", bien qu'on le ressente ainsi, c'est qu'il est trop pesant, vécu seul et non de manière commune. Que ce soit par l'attente à laquelle sont sujets les précaires ou par l'individualisation des rapports au temps de travail dans le monde salarié, le quotidien devient saturé.

Si certains osent à dire que la précarité et ses temps «fractionnés " permettraient un détachement du travail et ouvriraient de nouveaux possibles d'émancipation ${ }^{71}$, il est trop fréquemment oublié, nous semble-t-il, de prendre la mesure des enjeux temporels dans leur relation à l'espace. S'il apparaît plausible dans une analyse théorique marxiste ou anarchiste de voir dans l'émiettement du temps de travail l'ouverture d'une brèche dans laquelle sombrerait la valeur travail, reste qu'il faut bien des espaces pour habiter ces temps nouveaux. Les mouvements sociaux en faveur de l'émancipation en témoignent à bien des égards. Alors que les revendications pour la réappropriation de l'espace public et la critique de la ville capitaliste ont longtemps été incarnées par le mouvement squat - et bien que celui-ci continue son activité - d'autres alternatives semblent être privilégiées aujourd'hui par les mouvements pour l'émancipation sociale et installent l'appropriation de l'espace dans une relation étroite à l'institution de temps nouveaux. Trois cas témoignent selon nous d'un intérêt de plus en plus prononcé pour la formulation d'un droit au temps qui résonne à la revendication du droit à la ville. Ce collectif est par ailleurs en train de se recomposer et il ne serait pas étonnant d'y voir, comme c'est le cas pour d'autres CREP en France, les revendications relatives au temps s'y faire plus précise. 


\section{Le temps de la fête contre le temps urbain marchand : le CREP à Caen}

14 De 2008 à 2011, la ville de Caen a vu sa politique urbaine perturbée par un Collectif de Réappropriation de l'Espace Public (CREP). Ce collectif organisait des projections et des débats sur les questions relatives à l'urbanisme sécuritaire et à la ségrégation sociospatiale. Regroupant différents collectifs ${ }^{72}$, les militants du CREP produisaient un discours critique sur la thèse de l'urbanisme criminogène et sur le fonctionnalisme, sur le mobilier urbain "à vocation disciplinaire", ainsi que des matériaux comme une cartographie de la vidéosurveillance sur le campus. Leurs journées d'actions consistaient en une fraude collective des transports en commun pour diffuser des informations sur les transports gratuits, une déambulation en vélo en occupant les voies circulatoires automobiles, des recouvrements de publicité, et à la nuit tombée étaient éteints les néons extérieurs des commerces. Au milieu de l'après-midi, une place était également occupée où se déroulaient différentes activités (table de presse militante, goûter à prix libre, concerts, jeux, graffitis à la craie). Ce collectif, porté principalement par des activistes des milieux radicaux, mais rassemblant plus largement lors des journées d'actions ${ }^{73}$, proposait une forme d'action directe à la frontière entre légalité et illégalité dans l'optique de "se réapproprier nos vi (ll) es pour repenser une société qui soit ce que nous décidons d'en faire et non pas une vi (ll) e où nous serions assujetties au rôle de consommateur-acteur $»^{74}$ et de " prendre le temps de se rencontrer, de papoter, d'échanger, de s'exprimer, de regarder, d'écouter, de dire "stop !" plutôt que de simplement s'y déplacer pour aller d'une vitrine à l'autre $»^{75}$. Au final, lors de ces journées, le CREP cherchait à proposer au temps urbain de retrouver le temps de la fête en sabotant la rythmique marchande de la ville capitaliste. L'intérêt porté aux transports en commun et aux mobilités traduit également une préoccupation importante ouverte sur les rythmes urbains et la critique du fonctionnalisme qui, dans une ville comme Caen a largement participé idéologiquement à la reconstruction d'après-guerre, a participé à produire un espace dans lequel la voiture allait prendre une place dominante ${ }^{76}$.

Aujourd'hui, si cette histoire du CREP est encore présente dans les initiatives militantes extra-parlementaires locales, le collectif est en reconstitution et se trouve porté par une nouvelle génération de militants inscrits dans une "filiation» directe avec l'ancienne génération. Les anciens militants du CREP ont d'ailleurs été appelés à témoigner de leurs expériences et à participer au nouveau projet. Celui-ci, suivant les mutations de l'urbain, de son temps et de son économie, s'oriente vers une attention plus spécifique sur les dispositifs de contrôle comme la vidéosurveillance, et face à la création d'un "Minatec Caennais", à la problématique des nouvelles technologies comme nouveaux outils de contrôle social et d'accélération de l'obsolescence programmée.

\section{L'espace de la grève permanente : la Maison de la Grève à Rennes}

16 En octobre 2010, à Rennes, durant le mouvement contre la réforme des retraites, s'est ouvert une Maison de la Grève. La création de cet espace devait permettre de donner une base ouverte à tous ceux qui « résistent à la rationalité économique qui chaque jour écrase un peu plus " par une plateforme de coordination des piquets de grève, de 
blocage des "points névralgiques de l'économie " et de constitution de caisses de grèves pour soutenir l'effort de grève. Cette occupation illégale d'anciens locaux de la CFDT devait « rendre impossible tout retour à la normale » et donner « la force de tout arrêter " au-delà des «identités et des corporatismes " ${ }^{77}$. La particularité de cette tentative réside dans la volonté de maintenir la grève comme suspension du déterminisme temporel de l'économie qui s'appuie sur l'expropriation du temps. Suite à l'expulsion du lieu squatté illégalement, le 2 décembre, un cortège d'environ trois cents personnes s'est rendu au couvent des jacobins, ancien lieu religieux transformé en caserne au XVIIIe siècle puis vendu à Rennes métropole en 2002, afin de se réunir et discuter des perspectives pour la lutte en cours. Au sein du couvent se trouvait une exposition sur le futur centre des congrès projeté par Rennes Métropole. Les activistes ont alors saccagé l'exposition, puis furent accusés de s'en être pris au lieu même. Un tract d'éclaircissement est publié le 14 décembre dans lequel la critique de la métropolisation s'ajoute aux revendications de grève : «C'est uniquement l'exposition qu'elle contenait qui a été malmenée, ce qui fait une différence de poids quand on sait de quelle "exposition" il s'agissait. Rien d'artistique, là-dedans. Rien qu'un hymne à la conception affairiste de la politique. Un bête porno d'anticipation pour inconditionnelles du diktat de l'économie et de l'urbanisme sécuritaire ${ }^{78}$. Effectivement, les individus qui se retrouvent dans cette lutte portent alors une réflexion pointue sur les enjeux de luttes en termes d'espaces et de temps. Et cette perspective théorique entraina les activistes vers l'orientation stratégique de la location afin d'offrir un lieu pérenne: "Pour construire, au fil des temps, une force locale déterminée à vivre autre chose que le capitalisme [...], la Maison de la Grève n'est qu'une ébauche, un commencement ici et maintenant d'autres possibilités. [...] Nous ne voulons plus laisser notre quotidien au hasard de ce monde. Nous voulons nous en ressaisir collectivement, partager et étendre des pratiques offensives. S'organiser contre le réaménagement de nos espaces, soutenir les grèves, imaginer des actions en dehors des mouvements sociaux, tout en se liant avec des initiatives d'ailleurs. Être un lieu d'où partir et où revenir, un lieu pour se projeter collectivement ${ }^{79}$.

Rejetant le qualificatif de "militants" qu'ils considèrent comme une catégorie appartenant au mouvement social et aux formes organisationnelles traditionnelles qui $\mathrm{y}$ sont liées ${ }^{80}$, ceux qui portent le projet $\mathrm{y}$ voient une rencontre des subjectivités traversées par le désir commun d'en finir avec l'existant, tension théorique qui traverse les milieux autonomes du négrisme jusqu'à l'anarchisme insurrectionniste: "Pour apprendre ce qu'est la liberté, il n'y a pas d'autres manières que de l'expérimenter. Et pour l'expérimenter, il faut avoir le temps et l'espace nécessaires. [...] D'un côté il y a l'existant, avec ses habitudes et ses certitudes. Et de certitudes, ce poison social, on en meurt. D'un autre côté, il y a l'insurrection, l'inconnu qui surgit dans la vie de tous. Le possible début d'une pratique exagérée de la liberté ${ }^{81}$.

18 Au-delà du slogan des intermittents et précaires de Paris qui soutient que «nous avons besoin de lieux pour habiter le monde ", la Maison de la Grève par l'orientation stratégique locative soutient quelque part qu'il y a besoin de temps autant que d'espaces pour habiter les possibles alternatives à l'économie capitaliste et son urbanisme. La revendication d'autonomie y est forte et s'expérimente quotidiennement sur différents registres mêlés (autonomie dans la production des savoirs et dans la production matérielle) et différents champs (four à pain, mécanique, brasserie et mise en fut, cantines populaires, paysannerie et meunerie en lien avec la ZAD de Notre- 
Dame-des-Landes, informatique et protection numérique, cartographie des flux économiques et des enjeux stratégiques de la conflictualité politique, séminaire sur l'histoire de l'art et des luttes, séminaire de philosophie... etc.). Il n'est alors pas étonnant de voir, face à la répression accrue contre les occupations illégales, des formes associatives offensives s'affirmer dans différentes villes de France par la stratégie locative qui vise à inscrire les espaces appropriés dans le temps long.

\section{«DIY spaces » ou des espaces permanents pour l'autonomie contre- culturelle : Londres}

19 Le durcissement des lois de criminalisation des occupations n'est pas qu'un phénomène français. En Angleterre, une loi datant d'août 2012 établit des peines pouvant aller jusqu'à six mois de prison et 5000 livres d'amende pour l'occupation illégale d'un bâtiment. Longtemps connue pour être une capitale du squat sous la poussée de la spéculation et de la gentrification, la ville de Londres devient de plus en plus un terrain difficile pour l'appropriation de l'espace. Faisant face à ces difficultés, des activistes liés à la scène punk Do It Yourself - scène qui se rapproche d'une version contre-culturelle de l'anarchisme - ont décidé eux aussi de louer un local afin d'y mener des activités diverses. Le DIY Space For London est une salle de concert autonome, un bar associatif, une cantine, un atelier sérigraphie, un lieu de réunion, un espace de performance et d'expositions artistiques, etc. Se présentant comme une mise en acte des idées d'aide mutuelle et de coopérativisme, cet espace fonctionne sur les dons et des soirées grâce auxquels les activistes, organisés en assemblée, peuvent rassembler les fonds nécessaires au paiement du loyer de ces $300 \mathrm{~m}^{2}$. Ben, un des fondateurs, confie qu'il leur ait apparu fondamental, paradoxalement à leurs idées contestataires, d'être le plus en accord avec la loi. Pour les activistes du DIY Space for London, la légalité est un moyen de ne pas donner à la police l'opportunité de déstabiliser l'entreprise politique. Défendant un espace de pratiques autonomes et d'autonomisation, Mike, un adhérent ancien squatteur, explique qu'il préfère donner une cotisation au DIY Space afin d'avoir un espace pérenne et sortir de la précarité des espaces inhérente aux squats. Pour lui, les deux logiques ne s'opposent pas, au contraire. Depuis le DIY Space, autonome et pérenne, peuvent partir des initiatives plus offensives et peuvent s'expérimenter d'autres modes de délibération et de rencontre. Les activistes qui s'y retrouvent cherchent à la fois l'autonomie de l'espace, autonomie dont la modalité temporelle semble particulièrement importante: il y a besoin de temps pour prendre collectivement des décisions et éviter une «bureaucratisation » de l'organisation du lieu.

\section{Conclusion : conflits dans les temporalités, recherche du temps long}

La perspective sociohistorique permet donc de comprendre les logiques qui conduisent les démarches collectives de luttes contemporaines hors des organisations traditionnelles ou des associations liées à l'économie sociale et solidaire. Celles-ci semblent en effet innover en puisant dans l'histoire des luttes et en reformulant une grammaire normative nouvelle de l'instituant qui, dans leur forme même, maintiennent un certain niveau de conflictualité avec l'institué. Le paradoxe 
intéressant réside dans le compromis "éthique " qui tend à accepter le légalisme jusqu'à un certain degré, en ce sens qu'il permettrait de se doter de temps et d'espaces pour expérimenter dans le cadre de la loi des initiatives qui troublent pourtant de manière indirecte l'ordre public. Néanmoins, toutes ses expérimentations ne se défendent pas de la position légaliste, bien au contraire. Il s'agit d'une instrumentalisation des cadres légaux permettant d'obtenir a minima une stabilité organisationnelle inscrite dans des temps et des espaces pérennes, d'où pourraient émerger ensuite des dynamiques augmentant le degré de conflictualité. Ces espaces de luttes permettent également, dans le cadre de la répression de ne pas être soumis à l'urgence du temps juridique et de pouvoir organiser des évènements de soutien pour nourrir les caisses de solidarité.

21 Si les problématiques du temps et de l'espace sont étroitement liées, tant dans la production de l'espace capitaliste que dans les luttes contre celle-ci, les dynamiques collectives présentées ici s'interrogent également sur la façon dont «se donner le temps» permet aussi une formation extra-universitaire aux cadres théoriques nécessaires à la reformulation critique sociale cohérente avec la situation historique. En effet, critiques de la domination et du savoir légitime, nombreux et nombreuses sont les activistes à user de concepts et d'auteurs partagés avec le monde académique. Entre par exemple (parmi d'autres) les références à Henri Lefebvre au sein du CREP, les ateliers Spinoza de la maison de la Grève, les textes marqués de foucaldisme à Londres, ces expériences se présentent également comme des temps de formation qui rendent poreuse la distinction entre le savoir légitime et le savoir profane, ou en tout cas interroge la validité de la domination de l'un sur l'autre.

Il est important de remarquer également comment les orientations pratiques s'attachent à recréer des espaces-temps communs contre la dépossession des espaces et la saturation du temps social. La fabrique du commun observée confirme en partie l'hypothèse de la saturation, en ce sens que le désir d'appropriation d'un espace conduit en amont et en aval à l'émergence de temps communs et de registres d'actions collectives. Enfin, toutes les expériences mentionnées pour exemplifier l'analyse théorique sont attachées au retour de la fête, d'une fête ascendante et non pas déterminée par un calendrier extérieur. La fête est un moment de convivialité pour certains, un moment cathartique pour d'autres, qui permet à la fois de sortir des rapports sociaux quotidiens afin de créer des espaces-temps de rencontres des subjectivités. À ce titre, c'est bien la revendication d'un droit au temps qui rejoint celle du droit à la ville : la fête n'est pas nécessairement ce qui s'organise dans l'espace politique, mais bien ce qui en déborde, ce qui occupe l'espace marchand, le subvertit, dans un désir commun de se saisir de la ville et d'oublier le temps des marchands. La ville est encore et toujours le théâtre d'une révolution de la vie quotidienne qui n'a pas échoué, mais qui n'est tout simplement pas achevée. 


\section{BIBLIOGRAPHIE}

Ascher, F., 1995, Métapolis: Ou l'avenir des villes, Paris : Odile Jacob.

Benevolo, L., 1993, La Ville dans l'histoire européenne, Paris : Seuil.

Bertheleu, H., 2008, « Démocratie participative : entre gestion urbaine et citoyenneté », in Bertheleu H., Bourdarias, F. (dir.), Les constructions locales du politique, Tours : Presses universitaires François-Rabelais, 2008, p. 43-54.

Blum, L., 1936, « Léon Blum (1936) : « Nous sommes un Gouvernement de Front populaire » (6 juin 1936) - Histoire - Grands moments d'éloquence - Assemblée nationale », , En ligne : http:// www2.assemblee-nationale.fr/decouvrir-l-assemblee/histoire/grands-moments-d-eloquence/ leon-blum-1936-nous-sommes-un-gouvernement-de-front-populaire-6-juin-1936.

Boltanski, L., chiapello, È., 1999, Le nouvel esprit du capitalisme, Paris : Gallimard.

Bourdier, M., 2015, « Macron étrille les 35 heures, l'aile gauche du PS monte au créneau », Le Huffington Post, En ligne : http://www.huffingtonpost.fr/2015/08/28/35-heures-emmanuelmacron-aile-gauche-parti-socialiste_n_8052558.html.

Bourdieu, P., 2012, Sur l'Etat: cours au Collège de France (1989-1992), Paris : Seuil/Raisons d'agir. Boutinet, J.-P., 2004, Vers une société des agendas: une mutation de temporalités, Paris : Presses universitaires de France.

cingolani, P., 2012, Le temps fractionné: multiactivité et création de soi, Paris : Armand Colin. cingolani, P., 2014, Révolutions précaires: essai sur l'avenir de l'émancipation, Paris : La Découverte. corbin, A., 2000, «Temps des loisirs, espaces de la ville », Histoire urbaine, vol. $n^{\circ} 1, n^{\circ} 1, p$. 163-168.

corbin, A., 2001, L'avènement des loisirs: 1850-1960, Paris : Flammarion.

coutrot, T., 1998, «L'entreprise néo-libérale : la coopération forcée », in Coutrot, T., L'entreprise néolibérale, nouvelle utopie capitaliste?, Paris : TAP/Economie, p. 219-253.

crary, J., 2014, 24/7 le capitalisme à l'assaut du sommeil, Paris : Zones.

cultiaux, J., 2013, « L'argument de la « modernisation » dans la transformation des entreprises publiques : l'exemple de Belgacom », La nouvelle revue du travail [En ligne], 2, mis en ligne le 30 mars 2013, consulté le 11 septembre 2016 : https://nrt.revues.org/875.

Dumazedier, J., 1964, « Travail et loisir », in Friedmann, G., Naville, P. (dir.), Traité de sociologie du travail, Paris : Armand Colin, p. 341-366.

Durkheim, E., 1897, « La conception matérialiste de l'histoire », Revue philosophique, $\mathrm{n}^{\circ}$ XLIV, p. 645-651.

Durkheim, E., 1968, Les formes élémentaires de la vie religieuse : le système totémique en Australie, Paris : Presses universitaires de France.

Durkheim, É., 1981, Les règles de la méthode sociologique, Paris : PUF.

Durkheim, E., 2002, Friedrich Ratzel, Anthropogéographie un compte-rendu, Chicoutimi, J.-M. Tremblay. 
Durkheim, É., 2004, De la division du travail social, Paris : Presses universitaires de France. Foucault, M., 1993, Surveiller et punir: naissance de la prison, Paris : Gallimard. Freyssinet, J., 1998, « L'évolution du temps de travail : le déplacement des enjeux économiques », Droit Social, n 9/10, p. 752-759.

Friedmann, G., 1964, Le Travail en miettes, spécialisation et loisirs..., Paris : Gallimard.

Garnier, J.-P., 1980, « Espace marxiste, espace marxien », Espace géographique, vol. 9, n 4, p. 267-275.

Garnier, J.-P., Goldschmidt, D., 1978, La comedie urbaine: ou la cite sans classes, Paris : François Maspero.

Goetschel, P., Loyer, E., 2002, Histoire culturelle de la France de la belle époque à nos jours, Paris : Armand Colin.

Gourgues, G., TOPÇU, S., RUI, S., 2013, « Gouvernementalité et participation », Participations, n 6, p. $5-33$.

Grossin, W., 1969, Le travail et le temps, Paris : Anthropos.

Grossin, W., 1974, Les temps de la vie quotidienne, Paris - La Haye : Mouton.

Gurvitch, G., 1955, Determinismes sociaux et liberté humaine ; vers l'étude sociologique des cheminements de la liberté, Paris : Presses Universitaires de France.

Gurvitch, G., 1958, La multiplicité de temps sociaux, Paris : Centre de Documentation Universitaire. Habermas, J., 1978, L'Espace public : archéologie de la publicité comme dimension constitutive de la société bourgeoise, Paris : Payot.

Halbwachs, M., 1925, Les Cadres sociaux de la mémoire, par Maurice Halbwachs,..., Paris : F. Alcan. Harvey, D., 2010, Géographie et capital: vers un matérialisme historico-géographique, Paris : Syllepse. Harvey, D., 2012, Paris, capitale de la modernité, Paris : Les Prairies ordinaires. Harvey, D., 2008, Géographie de la domination, Paris : les Prairies ordinaires. Hubert, H., 1905, Etude sommaire de la representation du temps dans la religion et la magie, Paris : Imprimerie Nationale.

Jacquot, L., 2016, Travail, gouvernementalité managériale et néolibéralisme, Paris : L’Harmattan. Javeau, C., 2003, La société au jour le jour: écrits sur la vie quotidienne, Bruxelles : La lettre volée. Juan, S., 2015, « Le concept de routine dans la socio-anthropologie de la vie quotidienne », Espace populations sociétés. Space populations societies, En ligne : http://eps.revues.org/5935.

Lallement, M., 2003, Temps, travail et modes de vie, Paris : Presses universitaires de France.

Lallement, M., 2008, « Une antinomie durkheimienne... et au-delà », Temporalités. Revue de sciences sociales et humaines, En ligne : https://temporalites.revues.org/72.

Le Goff, J., 1960, «Au Moyen Âge : temps de l'Église et temps du marchand », Annales. Économies, Sociétés, Civilisations, vol. 15, n 3, p. 417-433.

Le Goff, J., 1977, Pour un autre Moyen Age: temps, travail et culture en Occident, 18 essais, Paris : Gallimard.

Lefebvre, H., 1958, Critique de la vie quotidienne : introduction, Paris : l'Arche. 
Lefebvre, H., 1968, La vie quotidienne dans le monde moderne, Paris : Gallimard.

Lefebvre, H., 1970, La révolution urbaine., Paris : Gallimard.

Lefebvre, H., 1975, L'idéologie structuraliste, Paris : Anthropos.

Lefebvre, H., 1981, Critique de la vie quotidienne : de la modernité au modernisme, Paris : L'Arche.

Lefebvre, H., 1986, Le retour de la dialectique, Paris : Messidor - Editions sociales.

Lefebvre, H., 2000, La production de l'espace, Paris : Anthropos.

Lefebvre, H., 2009, Le droit à la ville, Paris : Economica / Anthropos.

Lordon, F., 2010, Capitalisme, désir et servitude : Marx et Spinoza, Paris : la Fabrique.

Lourau, R., 1970, L'analyse institutionnelle, Paris : Éditions de Minuit.

Marx, K., 1957, Contribution a la critique de l'économie politique, Paris : Éditions sociales.

Marx, K., 1968, Misère de la philosophie, réponse à la philosophie de la misère de M. Proudhon., Paris :

Éditions sociales.

MarX, K., 1972, La Guerre civile en France: 1871, Paris : Éditions sociales.

Marx, K., 1976, Le Capital: critique de l'économie politique, Paris : Éditions Sociales.

Marx, K., 1980, Manuscrits de 1857-1858., Paris : Editions Sociales.

Marx, K., Engels, F., 1968, L’idéologie allemande, Paris : Éditions sociales.

Morin, E., 1962, L'esprit du temps: essai sur la culture de masse, Paris : Grasset.

Naville, P., 1963, Vers l'automatisme social ?: problèmes du travail et de l'automation, Paris : Gallimard.

Nicolas Le Strat, N., 2016, Le travail du commun, Saint Germain Sur Ille : Editions du Commun.

Noiriel, G., 1986, Les ouvriers dans la societe francaise: XIXe-XXe siecle, Paris : Seuil.

Raymond, H., 1959, « Hommes et dieux à Palinuro (observations sur une société de loisirs) », Esprit, $\mathrm{n}^{\circ}$ 6, p. 1030-1040.

Richez, J.-C., strauss, L., 2001, « Un temps nouveau pour les ouvrier : les congés payés

(1930-1960) ", in Corbin, A., L'avènement des loisirs. 1850-1960, Paris : Flammarion, p. 376-412.

Rioux, J.-P., sirinelli, J.-F., 2005, Histoire culturelle de la France 4, Le temps des masses : le vingtième siècle, Paris : Seuil.

Ripoll, F., 2005, « S'approprier l'espace... ou contester son appropriation? », Norois. Environnement, aménagement, société, $\mathrm{n}^{\circ} 195$, p. 29-42.

Rosa, H., 2010, Accélération: une critique sociale du temps, Paris : La Découverte.

seiller, P., 2014, « Sous-traitance et segmentations ouvrières dans la construction navale. », Sociologie, En ligne : https://sociologie.revues.org/2285.

Thompson, E. P., 1979, « Temps, travail, capitalisme industriel », Libre, n 79-5, p. 3-64.

vignet, J., 2014, L'ambivalence des associations. Du capitalisme associatif à l'espace public oppositionnel, Thèse de doctorat, Caen, Université de Caen Basse-Normandie. 


\section{NOTES}

1. Durkheim, É., 1981, Les règles de la méthode sociologique, Paris : PUF.

2. Hubert, H., 1905, Etude sommaire de la représentation du temps dans la religion et la magie, Paris : Imprimerie Nationale.

3. Halbwachs, M., 1925, Les Cadres sociaux de la mémoire, par Maurice Halbwachs,..., Paris : F. Alcan.

4. Gurvitch, G., 1955, Determinismes sociaux et liberté humaine; vers l'étude sociologique des cheminements de la liberté, Paris: Presses Universitaires de France; Gurvitch, G., 1958, La multiplicité de temps sociaux, Paris : Centre de Documentation Universitaire.

5. Friedmann, G., 1964, Le Travail en miettes, spécialisation et loisirs..., Paris : Gallimard.

6. Naville, P., 1963, Vers l'automatisme social?: problèmes du travail et de l'automation, Paris: Gallimard.

7. Morin, E., 1962, L'esprit du temps: essai sur la culture de masse, Paris : Grasset.

8. Lefebvre, H., 1975, L'idéologie structuraliste, Paris : Anthropos.

9. Grossin, W., 1969, Le travail et le temps, Paris : Anthropos ; Grossin, W., 1974, Les temps de la vie quotidienne, Paris - La Haye : Mouton ; Lallement, M., 2008, «Une antinomie durkheimienne... et au-delà ", Temporalités. Revue de sciences sociales et humaines, En ligne : https:// temporalites.revues.org/72.

10. Rosa, H., 2010, Accélération : une critique sociale du temps, Paris : La Découverte.

11. Boutinet, J.-P., 2004, Vers une société des agendas: une mutation de temporalités, Paris: Presses universitaires de France.

12. cingolani, P., 2012, Le temps fractionné: multiactivité et création de soi, Paris : Armand Colin.

13. Nicolas Le Strat, N., 2016, Le travail du commun, Saint Germain Sur Ille : Editions du Commun.

14. Bourdier, M., 2015, « Macron étrille les 35 heures, l'aile gauche du PS monte au créneau », Le Huffington Post, En ligne : http://www.huffingtonpost.fr/2015/08/28/35-heures-emmanuelmacron-aile-gauche-parti-socialiste_n_8052558.html.

15. Jacquot, L., 2016, Travail, gouvernementalité managériale et néolibéralisme, Paris : L'Harmattan.

16. crary, J., 2014, 24/7 le capitalisme à l'assaut du sommeil, Paris : Zones.

17. Lefebvre, H., 2000, La production de l'espace, Paris : Anthropos.

18. Trois espaces ont été enquêtés. Le Collectif de Réappropriation de l'Espace Public à Caen a été abordé en observation flottante, de sa constitution à sa dissolution, et l'étude est augmentée des observations et entretiens menées sur ce terrain par Julien Vignet dans le cadre de sa thèse de doctorat en sociologie (Vignet, 2014). La maison de la grève à Rennes a été abordée quant à elle de façon moins suivie. Nous avons participé à quelques manifestations présentées dans le récit, nous avons visité les deux expériences. Mais cela ne suffisant pas, nous avons choisi d'éplucher les textes pour interroger plus sérieusement le discours produit par les acteurs et combler le manque de régularité dans la phase d'observation. Le paragraphe abordant cette expérience a été discuté avec deux acteurs de cette expérience. Enfin, le DIY space, à Londres, a été observé à trois reprises, et face au manque de temps, deux entretiens formels ont été réalisé, ainsi que plusieurs discussions informelles lors des évènements. Ces cas, de par les méthodologies différentes qui les constituent, arborent bien plus une valeur figurative que proprement empirique. Néanmoins, ils nous semblent illustrer un champ d'action contemporain que l'on voit se mobiliser dans de nombreuses villes, et une stratégie d'action collective qui est de plus en plus discutée dans les milieux contestataires radicaux.

19. Bourdieu, P., 2012, Sur l'Etat: cours au Collège de France (1989-1992), Paris : Seuil/Raisons d'agir.

20. Thompson, E. P., 1979, « Temps, travail, capitalisme industriel », Libre, $n^{\circ}$ 79-5, p. 3-64.

21. Le Goff, J., 1960, "Au Moyen Âge: temps de l'Église et temps du marchand", Annales. Économies, Sociétés, Civilisations, vol. 15, n 3, p. 417-433 ; Le Goff, J., 1977, Pour un autre Moyen Age: temps, travail et culture en Occident, 18 essais, Paris : Gallimard. 
22. corbin, A., 2000, «Temps des loisirs, espaces de la ville », Histoire urbaine, vol. $\mathrm{n}^{\circ} 1, \mathrm{n}^{\circ} 1, \mathrm{p}$. 163-168.

23. Foucault, M., 1993, Surveiller et punir: naissance de la prison, Paris : Gallimard.

24. Harvey, D., 2012, Paris, capitale de la modernité, Paris : Les Prairies ordinaires.

25. Benevolo, L., 1993, La Ville dans l'histoire européenne, Paris : Seuil.

26. Habermas, J., 1978, L'Espace public : archéologie de la publicité comme dimension constitutive de la société bourgeoise, Paris : Payot, p. 38.

27. Lefebvre, H., 1970, La révolution urbaine., Paris : Gallimard, p. 25.

28. Lefebvre, H., 1970, La révolution urbaine., Paris : Gallimard.

29. Noiriel, G., 1986, Les ouvriers dans la societe francaise: XIXe-XXe siecle, Paris : Seuil ; Rioux, J.-P., sirinelli, J.-F., 2005, Histoire culturelle de la France 4, Le temps des masses : le vingtième siècle, Paris : Seuil.

30. Durkheim, E., 1897, « La conception matérialiste de l'histoire », Revue philosophique, $\mathrm{n}^{\circ} \mathrm{XLIV}, \mathrm{p}$. 645-651.

Durkheim, E., 1968, Les formes élémentaires de la vie religieuse: le système totémique en Australie, Paris : Presses universitaires de France ; Durkheim, É., 1981, Les règles de la méthode sociologique, Paris : PUF ; Durkheim, E., 2002, Friedrich Ratzel, Anthropogéographie un compte-rendu, Chicoutimi, J.-M. Tremblay ; Durkheim, É., 2004, De la division du travail social, Paris: Presses universitaires de France.

31. Peut-être faut-il rappeler qu'il ne s'agit pas d'institutions nécessairement spatialisées dans la définition socioanthropologique. On distinguera l'institution comme forme mentale et collective qui régit la vie sociale, de l'organisation qui incarne sa forme matérielle (Lourau, 1970).

32. Op. cit., XXII.

33. Durkheim, E., 1897, « La conception matérialiste de l'histoire », Revue philosophique, $\mathrm{n}^{\circ} \mathrm{XLIV}, \mathrm{p}$. 645-651.

34. Marx, K., 1957, Contribution a la critique de l'économie politique, Paris : Éditions sociales, p. 4.

35. Marx, K., Engels, F., 1968, L'idéologie allemande, Paris : Éditions sociales.

36. Marx, K., 1972, La Guerre civile en France: 1871, Paris : Éditions sociales.

37. Garnier, J.-P., 1980, «Espace marxiste, espace marxien », Espace géographique, vol. 9, $\mathrm{n}^{\circ}$ 4, p. 267-275.

38. Marx, K., 1980, Manuscrits de 1857-1858., Paris : Editions Sociales.

39. Harvey, D., 2008, Géographie de la domination, Paris : les Prairies ordinaires.

40. Marx, K., 1968, Misère de la philosophie, réponse à la philosophie de la misère de M. Proudhon., Paris : Éditions sociales, p. 64.

41. Marx, K., 1976, Le Capital: critique de l'économie politique, Paris : Éditions Sociales.

42. Ripoll, F., 2005, "S'approprier l'espace... ou contester son appropriation?", Norois. Environnement, aménagement, société, $\mathrm{n}^{\circ} 195$, p. 29-42.

43. Durkheim, E., 2002, Friedrich Ratzel, Anthropogéographie un compte-rendu, Chicoutimi, J.-M. Tremblay.

44. Blum, L., 1936, « Léon Blum (1936) : « Nous sommes un Gouvernement de Front populaire » (6 juin 1936) - Histoire - Grands moments d'éloquence - Assemblée nationale », , En ligne : http:// www2.assemblee-nationale.fr/decouvrir-l-assemblee/histoire/grands-moments-d-eloquence/ leon-blum-1936-nous-sommes-un-gouvernement-de-front-populaire-6-juin-1936.

45. Richez, J.-C., strauss, L., 2001, «Un temps nouveau pour les ouvrier: les congés payés (1930-1960) », in Corbin, A., L'avènement des loisirs. 1850-1960, Paris : Flammarion, p. 376-412.

46. Dumazedier, J., 1964, « Travail et loisir », in Friedmann, G., Naville, P. (dir.), Traité de sociologie du travail, Paris : Armand Colin, p. 341-366. 
47. Goetschel, P., Loyer, E., 2002, Histoire culturelle de la France de la belle époque à nos jours, Paris : Armand Colin.

48. Lefebvre, H., 1981, Critique de la vie quotidienne : de la modernité au modernisme, Paris : L'Arche.

49. Lefebvre, H., 1958, Critique de la vie quotidienne : introduction, Paris : l'Arche, p. 41.

50. Op. cit., p. 86.

51. Raymond, H., 1959, « Hommes et dieux à Palinuro (observations sur une société de loisirs) », Esprit, $\mathrm{n}^{\circ}$ 6, p. 1030-1040.

52. corbin, A., 2001, L'avènement des loisirs : 1850-1960, Paris : Flammarion.

53. Ascher, F., 1995, Métapolis: Ou l'avenir des villes, Paris : Odile Jacob.

54. Lefebvre, H., 2009, Le droit à la ville, Paris : Economica / Anthropos.

55. À ce propos, plusieurs documents d'époque sont en ligne sur le site Dailymotion sur la chaîne du ministère du logement, de l'égalité des territoires et de la ruralité. On peut encore voir, dans une ville comme Caen, les différences notables entre la reconstruction d'un centre-ville plus hausmannien, et aux abords de la vieille ville, une banlieue fonctionnaliste.

56. Lefebvre, H., 1970, La révolution urbaine., Paris : Gallimard.

57. Lefebvre, H., 1986, Le retour de la dialectique, Paris : Messidor - Editions sociales, p. 160.

58. Javeau, C., 2003, La société au jour le jour: écrits sur la vie quotidienne, Bruxelles : La lettre volée.

59. Durkheim, É., 1981, Les règles de la méthode sociologique, Paris : PUF, XXIII.

60. Juan, S., 2015, "Le concept de routine dans la socio-anthropologie de la vie quotidienne", Espace populations sociétés. Space populations societies, En ligne : http://eps.revues.org/5935.

61. Op. cit., p. 147.

62. Harvey, D., 2010, Géographie et capital : vers un matérialisme historico-géographique, Paris : Syllepse, p. 245-248.

63. Garnier, J.-P., Goldschmidt, D., 1978, La comédie urbaine : ou la cite sans classes, Paris : François Maspero, p. 30-31.

64. Op. cit. p. 79.

65. Garnier, J.-P., Goldschmidt, D., 1978, La comédie urbaine: ou la cite sans classes, Paris : François Maspero, p. 141-163.

66. Gourgues, G., TOPÇU, S., RUI, S., 2013, « Gouvernementalité et participation », Participations, ${ }^{\circ}$ 6, p. 5-33.

67. coutrot, T., 1998, "L'entreprise néo-libérale: la coopération forcée », in Coutrot, T., L'entreprise néolibérale, nouvelle utopie capitaliste?, Paris: TAP/Economie, p. 219-253 ; Lordon, F., 2010, Capitalisme, désir et servitude : Marx et Spinoza, Paris : la Fabrique.

68. Boltanski, L., chiapello, È., 1999, Le nouvel esprit du capitalisme, Paris : Gallimard.

69. cultiaux, J., 2013, «L'argument de la « modernisation » dans la transformation des entreprises publiques: l'exemple de Belgacom », La nouvelle revue du travail [En ligne], 2, mis en ligne le 30 mars 2013, consulté le 11 septembre 2016 : https://nrt.revues.org/875. Nous voyons, à l'université par exemple, au nom de «la communauté universitaire» - concept abstrait qui regroupe aussi bien les personnels techniques, les vacataires, les étudiants, les enseignants chercheurs et les administratifs -, des appels récurrents au "vivre ensemble » et à "l'effort collectif » afin de maintenir " une offre attractive ». Ainsi, chaque collectif ou syndicat qui se mobilise autour de revendications spécifiques (signature des contrats de travail et paiement mensualisé) à un statut (par exemple ici les vacataires) et menace de grève (ne pas assurer les cours) est soumis à un discours de délégitimation de l'action collective car ils nuiraient à l'échelle du département, puis de l'UFR, puis du campus, au bon fonctionnement et se lèveraient non contre l'administration, mais contre l'ensemble de la communauté.

70. seiller, P., 2014, "Sous-traitance et segmentations ouvrières dans la construction navale. ", Sociologie, En ligne : https://sociologie.revues.org/2285. 
71. cingolani, P., 2012, Le temps fractionné: multiactivité et création de soi, Paris : Armand Colin ; cingolani, P., 2014, Révolutions précaires: essai sur l'avenir de l'émancipation, Paris: La Découverte.

72. Clan du néon, transports gratuits, vélorution, collectif caennais contre la pub.

73. vignet, J., 2014, L'ambivalence des associations. Du capitalisme associatif à l'espace public oppositionnel, Thèse de doctorat, Caen, Université de Caen Basse-Normandie.

74. «Luttons contre le tout sécuritaire », Caen, Mars 2011

75. « Réponse aux mauvais choix de Copenhague », Caen, Décembre 2009

76. Lefebvre, H., 1968, La vie quotidienne dans le monde moderne, Paris : Gallimard.

77. « Ouverture de la maison de la grève », Rennes, 27 octobre 2010

78. « à propos de l'expulsion de la maison de la grève et de ses suites ", Rennes, 14 décembre 2010

79. « texte de présentation de la Maison de la Grève », Rennes, 26 janvier 2012

80. Le milieu autonome est, en partie et en localité, influencé théoriquement par certains textes comme L'Appel, dans lequel nous pouvons lire : « Nous désertons l'activisme. Sans oublier ce qui fait sa force : une certaine présence à la situation. Une aisance de mouvement en son sein. Une façon d'appréhender la lutte, non par l'angle moral ou idéologique, mais par l'angle technique, tactique. Le vieux militantisme donne l'exemple inverse. Il y a quelque chose de remarquable dans l'imperméabilité des militants aux situations ». Le texte intégral est disponible ici : http:// rocbo.lautre.net/poleis/Tiqqun/appel.html

81. «A couteaux tirés avec l'existants, ses défenseurs et ses faux critiques », traduit de la brochure italienne Ai ferri corti con l'esistente, i suoi difensori e i suoi falsi critici. Texte intégral en ligne ici : http://www.non-fides.fr/?A-couteaux-tires-avec-l-Existant

\section{RÉSUMÉS}

Cet article tend à exposer dans une démarche sociohistorique les mutations du temps social à travers une approche croisée des champs de l'urbain, du travail et de la vie quotidienne. Cette approche sociohistorique permet de rendre compte, à partir du passé, des mutations en cours. Défendant l'hypothèse d'une saturation du temps social entendue comme un désynchronisation des rythmes du travail et de l'urbain, l'auteur cherche également à dépasser la simple analyse des nouveaux rapports de domination et d'aliénation temporels à partir de cas figuratifs présentant des expériences contemporaines de résistance portant une double critique de la production de l'espace capitaliste et de la dépossession du temps. C'est l'occasion de voir, dans des démarches extra-institutionnelles, les adaptations des pratiques et pensées de l'émancipation qui répondent à ces mutations de la domination et de l'aliénation par des stratégies renouvelées. De ces expériences interrogeant le droit à la ville et le droit au temps dans leur relation dialectique, émerge l'hypothèse que la révolution de la vie quotidienne n'a pas échouée, mais qu'elle n'est peut-être pas achevée.

\section{INDEX}

Mots-clés : temps social, Henri Lefebvre, fonctionnalisme urbain, droit au temps, droit à la ville 